\title{
AN APPLICATION OF THE CALCULUS OF VARIATIONS IN THE LARGE TO THE EQUATIONS OF NONLINEAR ELASTICITY ${ }^{1}$
}

\author{
BY MELVYN S. BERGER
}

Communicated by E. Isaacson, January 25, 1967

The partial differential equations associated with many problems in elasticity are distinguished from other equations of mathematical physics by their nonlinearity, ellipticity and variational character. Generally a study of the totality of solutions of the boundary value problem associated with this system of higher order equations is required. The object of this note is to show, by example, that these problems are often mathematically tractable by the combined use of modern regularity theory for elliptic equations and the nonlinear invariants of the associated multiple integral variational problem.

In this note we study the problem of post-buckling behavior of thin elastic structures as defined by Von Karman's equations. In an earlier note [4], the author, together with $\mathrm{P}$. Fife, studied this problem for small deflections of thin plates. Here we outline how this work can be extended to study large deflections of plates and curved elastic structures, (i.e. shells).

Mathematically the associated variational problem requires us to study the critical points of a smooth functional on an infinite dimensional smooth manifold. This manifold arises naturally from the physical problem as a surface of "constant internal energy." This point of view enables us to apply the previous studies of nonlinear elliptic eigenvalue problems [5], [6] and Ljusternik-Schnirelmann category $[8],[10]$ to these problems.

Previous studies of the mathematical aspects of the buckling of thin elastic structures have been studied by Friedrichs [7], Reiss [9], Vorovich [11] among others. The results obtained here are closely related to the numerical studies of Bauer and Reiss [2]; [3]. The author is grateful to Professor E. Reiss for many helpful suggestions in connection with this work as well as suggesting the example of $\S 5$.

1. The boundary value problem and its variational reformulation for a clamped plate. Let $\Omega$ be a bounded domain in $R^{2}$ with boundary $\partial \Omega$ consisting of a finite number of arcs on each of which a tangent

1 This research was partially supported by: U. S. Army Research Office (Durham) DA-ARO-31-124-D365 and NSF Grant GP 3904. 
rotates continuously. Defined over $\Omega$ we consider the following system of partial differential equations and boundary conditions:

$$
\begin{aligned}
\Delta^{2} f & =-[u, u], \\
\Delta^{2} u & =\lambda[F, u]+[f, u], \\
u & =u_{x}=u_{y}=f=f_{x}=f_{y}=0 \quad \text { on } \partial \Omega
\end{aligned}
$$

where $[f, g]=f_{x x} g_{y y}+f_{y y} g_{x x}-2 f_{x y} g_{x y}, \Delta^{2}$ denotes the biharmonic operator and $F(x, y)$ is a given smooth function.

The physical significance of these equations is stated in [4].

We denote by $W_{2,2}(\Omega)$ the collection of all functions whose derivatives of all orders up to and including two lie in $L_{2}(\Omega) ; W_{2,2}(\Omega)$ is a Hilbert space with respect to the inner product

$$
(u, v)_{2,2}=\sum_{|\alpha|=2}\left(D^{\alpha} u, D^{\alpha} v\right)_{L_{3}(\Omega)} .
$$

$\dot{W}_{2,2}(\Omega)$ is the closure of $C_{0}^{\infty}(\Omega)$ in $W_{2,2}(\Omega)$. We define the following operators mapping $\dot{W}_{2,2}(\Omega)$ into itself:

$$
\begin{aligned}
(B(u, v), \phi) & =\int_{\Omega}[u, v] \phi \text { for } \phi \in C_{0}^{\infty}(\Omega), \\
L u & =B(u, F), \\
T u & =B(u, B(u, u)) .
\end{aligned}
$$

These operators $B, L$ and $T$ are well defined on $C_{0}^{\infty}(\Omega)$ and can be extended to $\dot{W}_{2,2}(\Omega)$ by means of the divergence form of the differential expression $[f, g]$, the Sobolev Imbedding Theorem and the Riesz representation theorem for linear functionals on $\dot{W}_{2,2}(\Omega)$, as in $[5$, Part II].

We now construct a variational principle for the system (1), (2) by considering the functionals

$$
\begin{aligned}
& \Phi_{1}(u)=\frac{1}{2}\|u\|^{2}+\frac{1}{4}(T(u), u), \\
& \Phi_{2}(u)=\frac{1}{2}(L u, u) .
\end{aligned}
$$

We note that the functional $\Phi_{2}(u)$ is weakly continuous on $\dot{W}_{2,2}(\Omega)$.

For each fixed positive number $R$, we define an energy surface as the infinite dimensional manifold

$$
\partial A_{R}=\left\{u \mid u \in W_{2,2}(\Omega), \Phi_{1}(u)=R\right\} .
$$

Theorem 1 (A Regularity Theorem). Any smooth solution of the system (1), (2) can be obtained as a critical point of the functional $\Phi_{2}(u)=\frac{1}{2}(L u, u)$ over the infinite dimensional Hilbert manifold $\partial A_{R}$. 
Conversely any such critical point determines a smooth solution of the system (1), (2) in $\Omega$ and at all sufficiently smooth portions of $\partial \Omega$.

The proof of this result follows from Theorem 1 of [4] which in turn is based on Agmon [1] and the fact that the Euler-Lagrange equations of the constrained variational problem yield generalized solutions of the system (1), (2). It is important for our treatment that the variational problem can be expressed entirely in terms of the function $u(x, y)$. Once $u(x, y)$ is obtained $f(x, y)$ is uniquely determined by virtue of the first equation of the pair (1) and the boundary conditions (2).

2. The infinite dimensional manifold $\partial A_{R}$. In order to determine the qualitative nature of the solutions of (1), (2) we begin by analyzing the topological structure of the manifold $\partial A_{R}$. This structure, in turn, is determined by the properties of $T$.

LemMa 1. Let the operator $T$ be defined as in $\$ 1$, then

(i) For any scalar $\sigma, T(\sigma u)=\sigma^{3} T(u)$;

(ii) $T$ maps weakly convergent sequences into strongly convergent sequences;

(iii) $(T u, u) \geqq 0$;

(iv) For $u \in C^{2}(\Omega) \cap C_{0}(\bar{\Omega}),(T u, u)=0$ if and only if $u=0$;

(v) $T$ is a variational operator, i.e. the Gateaux derivative of $\frac{1}{4}(T u, u)$ in the direction $v$ is $(T u, v)$ for every $v \in \dot{W}_{2,2}(\Omega)$;

(vi) $T$ is Frechet differentiable;

(vii) $\|T u-T v\| \leqq K_{0}\left(\|u\|^{2}+\|v\|^{2}\right)\|u-v\|$, where $K_{0}$ is some constant independent of $u$ and $v$.

Proof. Property (i) is a consequence of the definition of $T$. Properties (ii) and (vii) follow from Sobolev's Imbedding Theorem. By virtue of the divergence form of the expression $[f,[g, h]]$, we obtain

$$
(T u, u)=\|B(u, u)\|^{2} .
$$

Thus we obtain property (iii). The proof of property (iv) makes use of the additional fact that for sufficiently smooth $u$, the equation $B(u, u)=0$ reduces to the equation of Monge-Ampere type

$$
w_{x x} w_{y y}-w_{x y}^{2}=0
$$

Properties (v) and (vi) follow from the symmetries of the expression

$$
\int_{\Omega}[f,[g, h]]_{\phi} \quad \text { for } f, g, h, \phi \in C_{0}^{\infty}(\Omega)
$$


Theorem 2. $\partial A_{R}$ is a $C^{2}$ Hilbert manifold, with the following properties:

(i) $\partial A_{R}$ is a bounded starlike set in $\dot{W}_{2,2}(\Omega)$,

(ii) $\partial A_{R}$ is not the boundary of a convex set,

(iii) $\partial A_{R}$ is homeomorphic to the boundary of the Hilbert sphere of radius $R, \partial \sum_{R}$, for each fixed number $R$, (by the natural mapping along rays through the origin),

(iv) $\partial A_{R}$ is symmetric with respect to the origin $u=0$,

(v) On $\partial A_{R},\|u\| \geqq \alpha>0$ where $\alpha$ is a constant independent of $u$.

These results are immediate consequences of Lemma 1 and the definition of $\partial A_{R}$. For example, to show $\partial A_{R}$ is a bounded set, we note that if $\phi_{1}(u)=R,\|u\| \leqq(2 R)^{1 / 2}$ by property (iii) of Lemma 1 .

3. Solutions of the nonlinear problem "in the large." In this section we study the solution of the system (1), (2) as a function of $\lambda$. In [4], it was shown that if we denote by $\lambda_{1}$, the smallest eignevalue of $u=\lambda L u$, the system (1), (2) has no solutions for $\lambda \leqq \lambda_{1}$, except the trivial solution $u=f=0$. To supplement this result we have the following:

Theorem 3 (A Sturm-Liouville Theorem). For each positive number $R$, the equations (1), (2) have a countably infinite number of distinct solutions $u_{n}(R) \in \partial A_{R}$ with associated eigenvalues $\lambda_{n}(R) \rightarrow \infty$. Each $u_{n}$ is characterized as a solution of the variational problem

$$
\sup _{[V]_{n}} \min _{V} \frac{1}{2}(L u, u)
$$

where $V \in \partial A_{R}$ and $[V]_{n}$ is an appropriate homotopy class of sets containing $V$.

The proof of this result can be ortained from the author's paper [5], Browder [6], Palais [8], or Schwartz [10].

4. Bifurcation theory. Here we show that from each eigenvalue of the selfadjoint eigenvalue problem $u=\lambda L u$, independent of multiplicity, there bifurcates a solution of the full nonlinear problem. To do this, we consider $\lim _{R \rightarrow 0} \lambda_{n}(R)$. We denote by $P^{\infty}$, the infinite dimensional real projective space obtained by identifying antipodal points of the sphere $\|u\|=1$, and by cat ${ }_{P_{\infty}} V^{\prime}$, the Ljusternik-Schnirelmann category of $V^{\prime}$ relative to $P^{\infty}$, (cf. [10]).

Lemma 2 (Generalized COURANT minimax PRINCIPLE). The eigenvalues $\lambda_{n}$ of the equation $u=\lambda L u$ can be characterized as follows:

$$
\left(\lambda_{n}\right)^{-1}=\sup _{\left[0^{\prime}\right]_{n}} \min _{V^{\prime}}(L u, u)
$$


where $V^{\prime}$ is a set $C P^{\infty}$ such that cat $_{P_{\infty}} V^{\prime} \geqq n$ and $\left[v^{\prime}\right]_{n}$ is the class of all such sets in $P^{\infty}$.

Theorem 4. From each eigenvalue of the equation $u=\lambda L u$ there $b i$ furcates at least one branch of nontrivial solutions of (1), (2). In particular from a simple eigenvalue there bifurcates exactly one such branch, and from an eigenvalue of multiplicity two there bifurcates at least two such branches.

The importance of this result is that each critical point, degenerate or nondegenerate, of the quadratic variational problem of Lemma 2 is stable under perturbation by the nonlinear functional $(T u, u)$. To prove the first part of the theorem we use the characterization of $\lambda_{n}(R)$ and $\lambda_{n}$ given in Theorem 3 and Lemma 2, to show $\lim _{R \rightarrow 0} \mid \lambda_{n}(R)$ $-\lambda_{n} \mid=0$. The qualitative study of solutions of small norm as a function of $\lambda$ was stated in [4]. At a double eigenvalue $\lambda_{0}$ of the linearized problem $u=\lambda L u$ we show that the solutions of small norm of the nonlinear problem can be reduced to the simple one-dimensional problem of ascertaining the number of real roots of an algebraic equation of degree 4 with real coefficients. This fact combines with the first part of the theorem to give the stated result.

5. Buckling of thin curved elastic structures. Although the mathematical phenomena associated with the buckling of curved elastic structures are known to differ considerably from those associated with plates, we indicate in this section that this fact is due, in large part, to the more subtle related variational problem. For simplicity, we consider the example of the buckling of an axially compressed cylindrical planel with initial constant curvature measured by the constant $K$. The associated Von Karman equations for this problem are

$$
\begin{gathered}
\Delta^{2} f=-\frac{1}{2}[u, u]+K u_{x x}, \\
\Delta^{2} u=-\lambda u_{x x}+[f, u]-K f_{x x}, \\
u=u_{x}=u_{y}=f=f_{x}=f_{y}=0 \quad \partial \Omega^{\prime} .
\end{gathered}
$$

Here $\Omega^{\prime}$ is a rectangular domain in $R^{2}$. (If $K=0$, these equations reduce to a special case of equations (1), (2).)

Once again the analogue of Theorem 1 is valid. However, in this case, $\partial A_{R}^{\prime}$, the associated infinite dimensional manifold is homeomorphic to a sphere for small values of $R$ and for large values of $R$. For such values of $R$, the Sturm-Liouville Theorem 3 holds although the proof requires an extra approximation argument. Finally the first part of the bifurcation Theorem 4 is valid. In this case, it is possible 
to have nontrivial solutions of small norm for $\lambda<\lambda_{n}$ and in the vicinity of $\lambda_{n}$.

\section{BIBLIOGRAPHY}

1. S. Agmon, The $L_{p}$ approach to the Dirichlet problem, Ann. Scuola Norm. Sup. Pisa 13 (1959), 405-448.

2. F. Bauer and E. Reiss, Nonlinear buckling of rectangular plates, J. Soc. Indust. Appl. Math. 13 (1965), 603-627.

3. - Post-buckling behaviour of axially compressed cylindrical panels, (to appear).

4. M. Berger and P. Fife, On Von Karman's equations and the buckling of a thin elastic plate, Bull. Amer. Math Soc. 72 (1966), 1006-1011.

5. M. Berger, $A$ Sturm-Liouville theorem for nonlinear elliptic partial differential equations, Ann. Scuola Norm. Sup. Pisa 20 (1966), 543-582.

6. F. Browder, Ljusternik-Schnirelmann category and nonlinear elliptic eigenvalue problems, Ann. of Math. 72 (1965), 459-477.

7. K. Friedrichs, On the minimum buckling load for spherical shells, Von Kraman Anniversay Volume, 1941 pp. 258-272.

8. R. Palais, Ljusternik-Schnirelmann theory on Banach manifolds, Topology 15 (1966), 115-132.

9. E. Reiss, Bifurcation buckling of spherical caps, Comm. Pure Appl. Math. 18 (1965), 65-82.

10. J. Schwartz, Generalizing the Ljusternik-Schnirelmann theory of critical points, Comm. Pure Appl. Math 17 (1964), 807-815.

11. I. Vorovich, Certain questions on the large deformation stability of shells, Soviet Physics Dokl. 3 (1958), 1032-1035.

Courant Institute of Mathematical Sciences, NEW YORK UNIVERSITY 